\title{
Radio source evolution from galaxy core to intracluster medium
}

\author{
Tamela Maciel and Paul Alexander \\ Astrophysics Group, Cavendish Laboratory, University of Cambridge, \\ J. J. Thomson Ave, Cambridge CB3 0HE, UK \\ email: tm419@cam.ac.uk
}

\begin{abstract}
There is mounting evidence that mechanical kinetic-mode AGN feedback is important in galaxy evolution, and in order to quantify this feedback, detailed models of radio source evolution are required. Self-similar analytic models exist for large powerful radio sources but the evolution of young precursor radio sources is not yet fully understood. In this talk we present a versatile dynamical and radiative model for young source evolution on sub-kiloparsec scales, which extends existing self-similar models into a more complete radio source evolutionary model. This semi-analytic model is successful in reproducing the strong spectral aging observed in compact symmetric objects.
\end{abstract}

Keywords. galaxies: jets - radiation mechanisms: non-thermal - galaxies: active

\section{Introduction}

Understanding the detailed evolution of radio-loud jets and cocoons as they propagate through the intragalactic and intracluster medium (IGM/ICM) is vital in order to quantify the importance of mechanical radio source feedback in galaxy and cluster evolution. On large intergalactic scales successful analytic models exist which determine the lifetime, growth, and energetics of classic double radio sources (e.g., Kaiser \& Alexander 1997; Kaiser et al. 1997). But to address kinetic-mode feedback on galactic scales, a new model of compact young source evolution is required to keep pace with the growing numbers of sub-kiloparsec scale observations and simulations of AGN feedback.

There are four commonly-used terms to classify compact radio sources (e.g., O'Dea 1998; Augusto et al. 2006). These are compact symmetric objects (CSOs), medium symmetric objects (MSOs), gigahertz peaked spectrum (GPS) sources, and compact steep spectrum (CSS) sources. CSOs exist on scales $\leqslant 1 \mathrm{kpc}$ while larger MSOs have sizes between $1 \mathrm{kpc}$ and $\sim 15 \mathrm{kpc}$. Measurements of the expansion velocities as well as spectral aging techniques find it likely that CSOs and MSOs form the precursors to the more mature Fanaroff and Riley type sources (FRI and FRII). The second two classifications come from a spectral view. GPS sources have sizes $<1 \mathrm{kpc}$ and a spectral peak around $1 \mathrm{GHz}$, while CSS sources have sizes between 1 and $\sim 15 \mathrm{kpc}$ and a spectral peak closer to $100 \mathrm{MHz}$. There is large overlap between these classifications in the sense that most CSOs are also GPS sources and that the MSO class contains the larger GPS objects and CSS objects. It is usually proposed that CSOs (GPS objects) evolve into MSOs (CSS objects), with the most powerful eventually becoming the classic FRIIs at large scales and the rest either shutting off completely or evolving into lower-luminosity, turbulent FRIs (e.g., Fanti et al. 1995; Alexander 2000; Kunert-Bajraszewska \& Labiano 2010). In this study we do not consider the duty cycle of CSOs, but rather model only those young sources that survive to become large-scale FRII sources. 
A dense core environment coincident with the sub-kiloparsec scales of CSOs will greatly affect how the source expands. A flat environment was considered by Kaiser \& Best 2007 and Kunert-Bajraszewska \& Labiano 2010, assuming self-similar expansion. Using a radiative model applied to both flat and falling environments, both studies predict strong synchrotron losses for young radio sources and provide instructive commentary for the young source regime. However their dynamics still assume a self-similar evolution, which is expected to break down on sub-kiloparsec scales (Falle 1991 and KA97). Therefore a more complex dynamical model to describe young source evolution is necessary.

A semi-analytic dynamical model for young source evolution which departs from selfsimilarity was developed by Alexander 2006, hereafter A06, and tracks radio source evolution from the point of cocoon formation up until jet reconfinement and the subsequent transition to self-similar expansion (Kaiser \& Alexander 1997, hereafter KA97). In this work we extend the dynamical model of A06 to include radiative evolution using the methodology outlined in Kaiser et al. 1997, hereafter KDA97, to track energy losses within the cocoon. We find that radiative losses are significant for young radio sources within a constant density environment. This work has been published in Maciel \& Alexander 2014, hereafter MA14, and the reader is referred there for more details.

\section{Young source evolution}

We consider the situation where a ballistic jet emerges from the central engine, expanding at relativistic speeds into an isotropic environment with a density gradient following a simplified King profile with a core density $\rho_{0}$,

$$
\rho_{\mathrm{x}}= \begin{cases}\rho_{0} & \text { if } r \leqslant a_{0} \\ \rho_{0}\left(\frac{a_{0}}{r}\right)^{\beta} & \text { if } r>a_{0}\end{cases}
$$

such that $\rho_{0} a_{0}^{\beta}$ becomes a characteristic quantity of the external medium. Here $a_{0}$ is the core radius of the host galaxy, typically around a few kiloparsecs in agreement with X-ray measures of galactic density profiles (e.g., Fukazawa et al. 2004; Kawakatu et al. 2008). Note that this profile differs from the simple power-law density profile adopted in KA97 and KDA97 to include a more realistic flat galaxy core.

The end of the jet shocks the IGM/ICM at the hotspot, and once the jet material becomes under-dense, a backflow of material at the hotspot inflates enormous cocoons filled with a plasma of particles and magnetic fields. A schematic diagram for young radio sources is shown in Figure 1, along with the length scales we discuss below. We use the following notation: subscripts $\mathrm{j}, \mathrm{h}, \mathrm{c}$, and $\mathrm{x}$ refer to the relevant property of the jet, hotspot, cocoon, and external environment, respectively.

The basic ingredients for a young radio source are assumed to be the same as for a larger self-similar source. The jet is characterized by its constant jet power $Q=\frac{1}{2} \dot{M} v_{\mathrm{j}}^{2}$, opening angle $\theta$, and mass transport rate, $\dot{M}$. The source expansion is governed by the external environment profile, $\rho_{\mathrm{x}}$. For the young source model, we assume the source is still confined within the galactic core and thus adopt a flat density profile, $\rho_{\mathrm{x}}=\rho_{0}$. From the above parameters we can write down a characteristic length scale,

$$
L_{1} \propto\left(\frac{\dot{M}^{3}}{\rho_{0}^{2} Q}\right)^{1 / 4} \equiv 2 \sqrt{2}\left(\frac{Q}{\rho_{0} v_{\mathrm{j}}^{3}}\right)^{1 / 2},
$$

where, following A06, we choose the $2 \sqrt{2}$ factor for convenience. Typical values for jet power $\left(Q=1.3 \times 10^{39} \mathrm{~W}\right)$, speed $\left(v_{\mathrm{j}}=c\right)$, and external density $\left(\rho_{0}=7.2 \times 10^{-22} \mathrm{~kg}\right.$ 

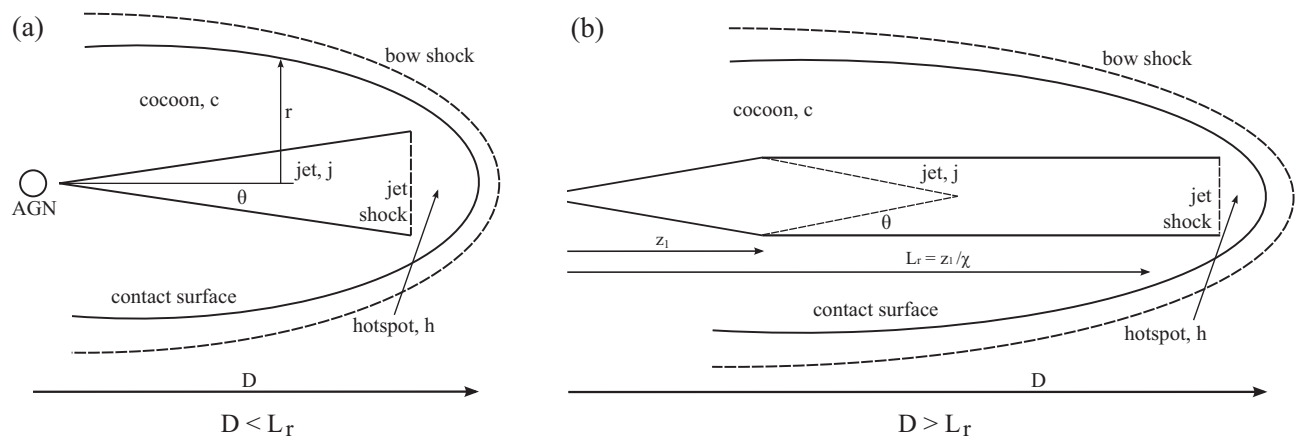

Figure 1. The model geometry and notation used in this work. Figure (a) is a snapshot schematic of a young radio source with an unconfined, conical jet and surrounding cocoon. Figure (b) is a snapshot diagram of a much older radio source, after the jet is fully reconfined by the cocoon pressure and evolution begins to follow a self-similar model. Diagrams not to scale.

$\mathrm{m}^{-3}$ ) give $L_{1}=24 \mathrm{pc}$. This length is tiny compared to the relevant scales of classical double sources, but here we seek to develop a young source model on galactic scales and this characteristic size cannot be ignored. Thus self-similar evolution is not expected for young sources. As discussed in A06, physically this length scale is similar to the scale when the jet density begins to fall below the constant external density, occurring at $L_{\mathrm{c}}$.

Our model considers three phases for full radio source evolution: (1) Beyond $L_{\mathrm{c}}$ the jet material is under-dense and a cocoon begins to inflate from the backflow of material at the hotspot. This onset of cocoon formation is where the young source model begins. The source will evolve according to the A06 model until the pressure in the cocoon is high enough compared to the falling sideways ram pressure of the jet in order to send an oblique reconfinement shock to the jet axis, at length scale $L_{\mathrm{r}}$ (Falle 1991). KA97 showed that this self-confinement process between the cocoon and the jet is crucial for self-similar evolution. The process of reconfinement depends upon the dynamics of the young jet and cocoon, and is discussed in more detail in MA14. (2) Following full reconfinement, the young source model ends and we join it to the self-similar model of KA97. The now selfsimilarly evolving source will continue to expand and radiate, initially in a flat density core environment and then (3) in a falling density environment until its end of life on megaparsec scales. After a typical FRII end of life around $t_{\text {end }}=10^{8} \mathrm{yr}$ (e.g. Bird et al. 2008; Antognini et al. 2012), we assume the jet switches off.

To plot a radiative evolutionary track on the well-known radio power-linear size $(P-D)$ diagram, expressions for how the source size, pressure, and volume evolve with age are required. These are derived in KA97, A06, and MA14, with the latter presenting the expressions for transitions between the three different phases.

\section{Radiative evolution}

To link the complete dynamical model (young source model plus self-similar evolution) to a radiative evolution we follow the approach of KDA97.

Synchrotron emission from the radio source cocoon is the dominant source of emission for the powerful radio sources considered in this model. The total power radiated from the cocoon per frequency per solid angle is

$$
P_{\nu}=\frac{\sigma_{\mathrm{T}} c u_{\mathrm{B}}}{6 \pi \nu} \gamma^{3} n(\gamma) V_{\mathrm{c}}
$$

where $u_{\mathrm{B}}$ is the cocoon magnetic field energy density, $\nu$ is the observing frequency, $\gamma$ 


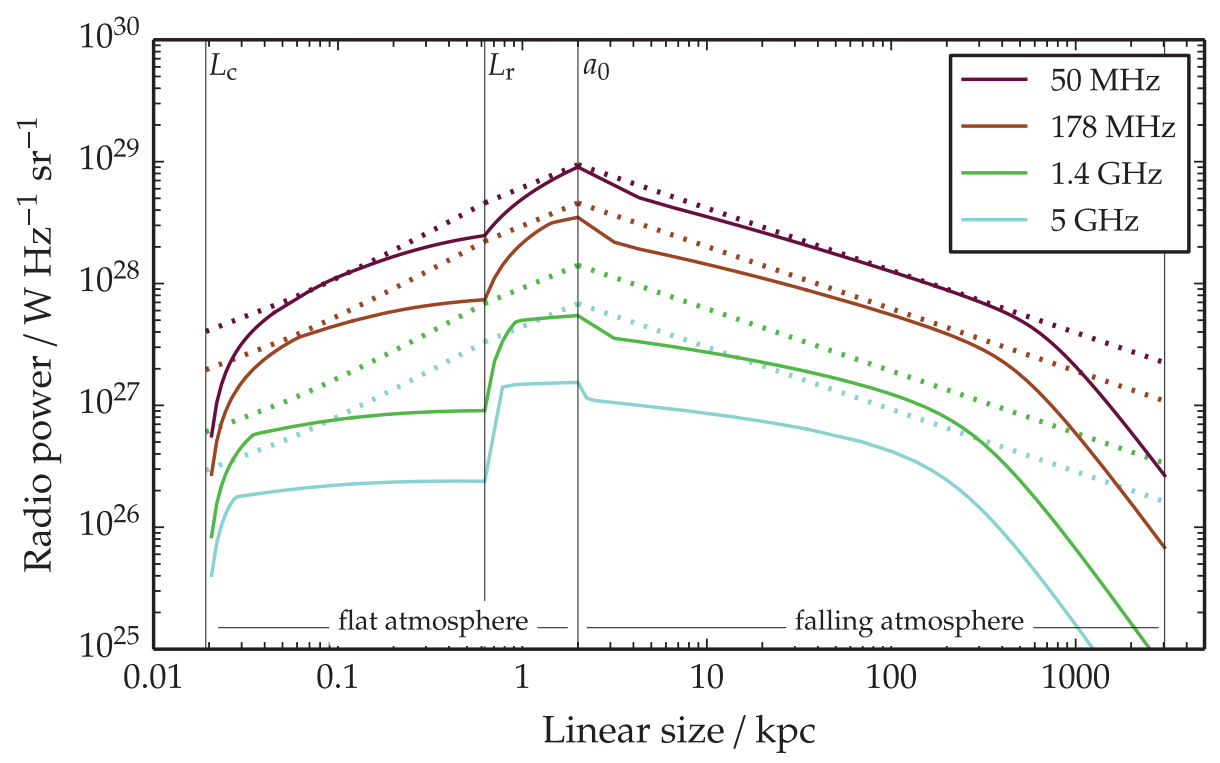

Figure 2. Radio source evolution through the $P-D$ diagram from first cocoon formation to end of life on intracluster scales, presented for observing frequencies, top to bottom, of $\nu_{\mathrm{obs}}=$ $50 \mathrm{MHz}$ (purple lines), $178 \mathrm{MHz}$ (brown lines), $1.4 \mathrm{GHz}$ (green lines), and $5 \mathrm{GHz}$ (blue lines). The dotted lines show evolution without radiative aging, the so-called 'loss-less' tracks. Here I use $Q=1.3 \times 10^{39} \mathrm{~W}, \theta=20^{\circ}$, and $z=2$. Other parameters given in MA14. Vertical lines mark the size boundaries between the three phases discussed in section 2 .

is the electron Lorentz factor, $n(\gamma)$ is the power-law distribution of electron energies, and $V_{\mathrm{c}}$ is the cocoon volume. By making the typical assumption of minimum energy conditions within the cocoon, we derive a simple 'loss-less' expression for radio power which has the usual $P_{\nu} \propto V_{\mathrm{c}} p_{\mathrm{c}}^{7 / 4}$ relation. This loss-less track through the $P-D$ diagram is shown as the dotted lines in Figure 2 for a range of observing frequencies.

Of course this loss-less model is simplistic given the nature of the expanding cocoon, and adiabatic expansion and radiative losses are significant. To include radiative losses we use the method of KDA97, keeping track of small volume elements, $\delta V_{\mathrm{c}}\left(t, t_{\mathrm{i}}\right)$, labeled by the source age, $t$, and the element's time of injection into the cocoon, $t_{\mathrm{i}}$.

For a population of electrons with a distribution over Lorentz factor following $n(\gamma) \mathrm{d} \gamma=$ $n_{0} \gamma^{-p} \mathrm{~d} \gamma$, energy losses are expressed via the rate of change of $\gamma$ (see MA14 for details). The subsequent expressions for the particle density evolution, the particle and magnetic co-evolution, and the expansion of individual volume elements given in KDA97 are all applicable to the radiative evolution of the complete dynamical model. Therefore the total radio power expression (per frequency per solid angle) is rewritten as

$$
P_{\nu}=\int_{t_{\min }(t)}^{t} \frac{\sigma_{\mathrm{T}} c u_{\mathrm{B}}(t)}{6 \pi \nu} \gamma^{3} n\left(t, t_{\mathrm{i}}\right) \delta V_{\mathrm{c}}\left(t, t_{\mathrm{i}}\right) \mathrm{d} t_{\mathrm{i}},
$$

whereby the radiating volume elements are integrated over the range of injection times from some minimum injection time, $t_{\min }(t)$, to the current source age. We define $t_{\min }(t)$ by when the Lorentz factor, $\gamma_{\mathrm{i}}$, of the injected electron at time $t_{\mathrm{i}}$ must approach infinity in order to still be radiating at time $t$. The integral for total radio power is not analytically tractable but can be numerically evaluated from first cocoon formation to end of source life to obtain the full path through the $P-D$ diagram for double radio sources. 


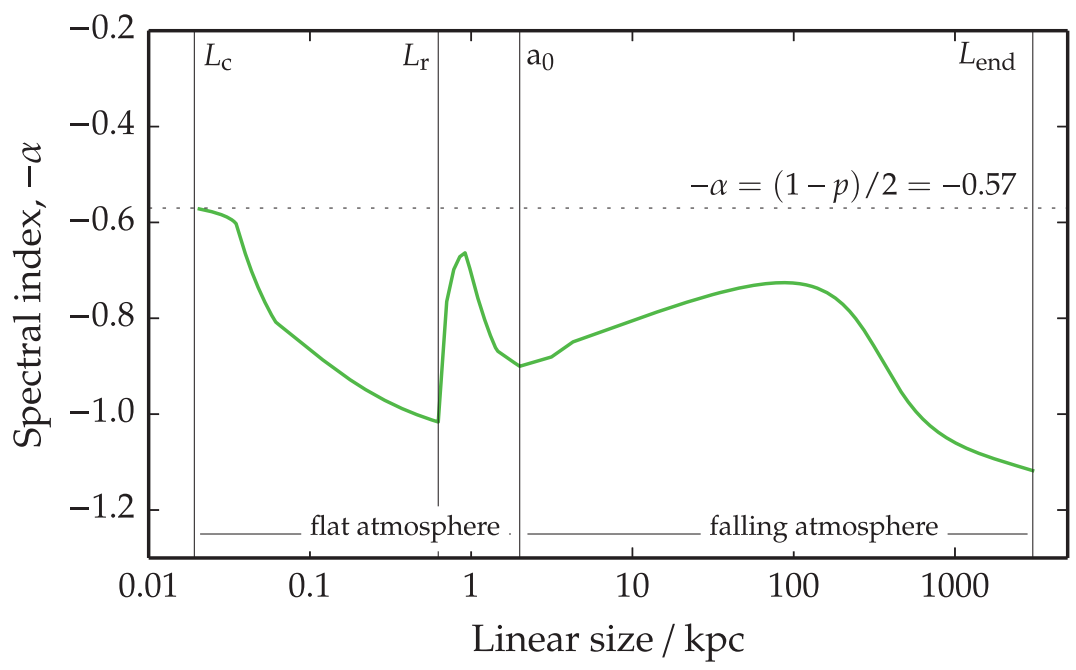

Figure 3. Cocoon spectral index, $-\alpha$, as it evolves with source size. $\alpha$ is measured between 178 $\mathrm{MHz}$ and $1.4 \mathrm{GHz}$, using the corresponding evolutionary tracks shown in Figure 2 and assuming a power-law synchrotron spectrum.

Figure 2 shows the evolutionary tracks for all three phases (solid lines), using equation 3.2. Phase 1 emission is evaluated using the dynamical expressions of A06 in a flat density environment. Phase 2 emission is evaluated using self-similar dynamics in a flat density environment. Phase 3 is evaluated using self-similar dynamics in a falling density environment. At the phase boundaries, the decaying emission from previous phases as well as the newly-injected material of the current phase are included in the total emission.

The sharp features at the boundaries in Figure 2 are caused by the fact that two different analytic models are matched across three regimes such that boundary discontinuities are inevitable. In reality, given a smooth reconfinement process and a smooth environmental profile, the luminosity evolution across the phase boundaries will also be smooth.

\section{Discussion of radiative aging in young sources}

On sub-kiloparsec scales, the evolutionary tracks of Figure 2 (solid lines) show significant departure from the corresponding loss-less tracks (dotted lines), particularly at high frequencies. This is indicative of radiative losses becoming important for young sources just before they transition to full self-similar evolution and before they leave the core environment. Strong radiative losses have been predicted before - see for instance the discussion and approximate $P-D$ track in Kaiser \& Best 2007. This work improves their model by including radiative losses for a more realistic, non-self-similar model of young source evolution.

We can further explore these radiative losses by estimating how the cocoon spectral index evolves with source size. This can be derived using the $P-D$ tracks at $\nu_{\mathrm{obs}}=178 \mathrm{MHz}$ and $1.4 \mathrm{GHz}$, assuming a power-law synchrotron spectrum, $S_{\nu} \propto \nu^{-\alpha}$. This spectral evolution, using the young model for $D<L_{\mathrm{r}}$ and the self-similar KDA97 model for $D \geqslant L_{\mathrm{r}}$, is shown in Figure 3. Although this is an approximate estimate of the overall cocoon spectral index, it does clearly predict a steepening of the cocoon spectra for young sources on sub-kiloparsec scales. Self-similar evolution in a falling atmosphere sees an initial flattening of the spectrum as radiative losses are minimal. The final spectral steepening occurs 
much later on scales of hundreds of kiloparsecs when inverse-Compton losses begin. At $100 \mathrm{kpc}$, we find an overall cocoon spectral index of -0.72 , in good agreement with typical measured spectral indices at this scale.

The scale of this spectral evolution matches observations of steep-spectrum CSOs and larger CSS sources, assuming that CSOs have sizes less than a kiloparsec and that CSS sources have kiloparsec sizes. For some source conditions, the rising spectral index at the beginning of phase 2 and phase 3 might translate to the growing awareness of flatspectrum MSOs (Augusto et al. 2006). Note that these scales change depending upon the particular jet power and environment density. But, in general, we find that young sources on sub-kiloparsec scales suffer from strong radiative losses and a steep spectral index. The process of reconfinement and the transition to a falling atmosphere both lead to a flattening of the spectral index, but IC losses at large scales steepen the total cocoon spectrum again. Kunert-Bajraszewska \& Labiano 2010 discuss in detail an evolutionary path from CSO to MSO to large-scale double sources with a very similar progression from steep-spectrum to flat to steep again at large scales, building on the work of Kaiser $\&$ Best 2007. Our radiative young source model agrees well with these earlier discussions.

\section{Summary}

In this talk we consider the evolution of the young radio source confined within its host galaxy and present a new radiative model which suggests strong spectral aging within the core environment. The young source model is linked to self-similar evolution on larger scales to form a complete dynamical and radiative analytic model of FRII source evolution from its first cocoon formation to its end of life on intracluster scales.

This complete model is more versatile than previous analytic models in that it incorporates a general history of the radio source which evolves according to different dynamical expressions in different external environments throughout its lifetime. Specifically in this work, we consider an evolution which links the two dynamical models of young source evolution (A06) and self-similar evolution (KA97), placed in a simplified King profile environment. With the addition of a continuous radiative history, we generate analytic evolutionary tracks through the $P-D$ plane which reproduce the observed spectral evolution of radio sources on both small and large scales.

\section{References}

Alexander, P. 2000, MNRAS, 319, 8

Alexander, P. 2006, MNRAS, 368, 1404

Antognini, J., Bird, J., \& Martini, P. 2012, ApJ, 756, 116

Augusto, P., Gonzalez-Serrano, J. I., Perez-Fournon, I., \& Wilkinson, P. N. 2006, MNRAS, 368, 1411

Bird, J., Martini, P., \& Kaiser, C. 2008, ApJ, 676, 147

Falle, S. A. E. G. 1991, MNRAS, 250, 581

Fanti, C., Fanti, R., Dallacasa, D., et al. 1995, A\&A, 302, 317

Fukazawa, Y., Makishima, K., \& Ohashi, T. 2004, PASJ, 56, 965

Kaiser, C. R. \& Alexander, P. 1997, MNRAS, 286, 215

Kaiser, C. R. \& Best, P. N. 2007, MNRAS, 381, 1548

Kaiser, C. R., Dennett-Thorpe, J., \& Alexander, P. 1997, MNRAS, 292, 723

Kawakatu, N., Nagai, H., \& Kino, M. 2008, ApJ, 687, 141

Kunert-Bajraszewska, M. \& Labiano, A. 2010, MNRAS, 408, 2279

Maciel, T. \& Alexander, P. 2014, MNRAS, 442, 3469

O'Dea, C. P. 1998, PASP, 110, 493 